\title{
The simultaneous oddball: Oddball presentation does not affect simultaneity judgments
}

\author{
Jordan Wehrman ${ }^{1}$
}

Published online: 15 January 2020

(C) The Psychonomic Society, Inc. 2020

\begin{abstract}
The oddball duration illusion describes how a rare or nonrepeated stimulus is perceived as lasting longer than a common or repeated stimulus. It has been argued that the oddball duration illusion could emerge because of an earlier perceived onset of an oddball stimulus. However, most methods used to assess the perceived duration of an oddball stimulus are ill suited to detect onset effects. Therefore, in the current article, I tested the perceived onset of oddball and standard stimuli using a simultaneity judgment task. In Experiments 1 and 2, repetition and rarity of the target stimulus were varied, and participants were required to judge whether the target stimulus and another stimulus were concurrent. In Experiment 3, I tested whether a brief initial stimulus could act as a conditioning stimulus in the oddball duration illusion. This was to ensure an oddball duration illusion could have occurred given the short duration of stimuli in the first two experiments. In both the first two experiments, I found moderate support for no onset-based difference between oddball and nonoddball stimuli. In Experiment 3, I found that a short conditioning stimulus could still lead to the oddball duration illusion occurring, removing this possible explanation for the null result. Experiment 4 showed that an oddball duration illusion could emerge given the rarity of the stimulus and a concurrent sound. In sum, the current article found evidence against an onset-based explanation of the oddball duration illusion.
\end{abstract}

Keywords Repetition effects $\cdot$ Decision making $\cdot$ Attentional capture

One minute is objectively 60 seconds. However, our subjective experience of 60 seconds can vary; 'time' can stretch and compress depending on whether we are sitting in traffic or on a rollercoaster. Recently, the 'oddball duration' illusion has garnered increased attention. The oddball duration illusion describes how a stimulus that occurs less frequently lasts subjectively longer than a repeated standard stimulus even if it has the same objective duration (Birngruber, Schröter, Schütt, \& Ulrich, 2017; Cai, Eagleman, \& Ma, 2015; Matthews \& Gheorghiu, 2016; New \& Scholl, 2009; Simchy-Gross \& Margulis, 2017; Tse, Intriligator, Rivest, \& Cavanagh, 2004; Wehrman, Wearden, \& Sowman, 2018). This effect occurs even with just two stimuli; if the second stimulus is a repeat of the preceding stimulus, it is perceived to last less time than if the second stimulus is different than the first (Birngruber et al., 2017; Birngruber, Schröter, \& Ulrich, 2014; Matthews, 2011, 2015; Matthews \& Gheorghiu, 2016; Skylark \&

Jordan Wehrman

Jordan.wehrman@students.mq.edu.au

1 Cognitive Science, Macquarie University Faculty of Science, Macquarie Park, Australia
Gheorghiu, 2017). In other words, for a nonrepeated stimulus to be perceived as lasting the same duration as a repeated stimulus, the objective duration of the nonrepeated stimulus must be shorter than the objective duration of the repeated stimulus. Investigating how the oddball duration illusion emerges serves to inform us on which features of our experience affect perceived duration, and how changes in perceived duration can occur.

Competing theories regarding how the oddball duration illusion occurs fall into three broad categories. Firstly, theories posit that perceived duration is a correlate of neural response size, such that a larger neural response results in a longer perceived duration (e.g., Eagleman, 2008; Eagleman \& Pariyadath, 2009; Pariyadath \& Eagleman, 2007; Schindel, Rowlands, \& Arnold, 2011). Secondly, perceived duration may be correlated with the amount of information processed in a given amount of time. Because a nonrepeated stimulus garners more attention, more information is captured in a given amount of time, resulting in a longer perceived duration (Tse, 2010; Tse et al., 2004; Ulrich, Nitschke, \& Rammsayer, 2006). Finally, a nonrepeated stimulus may actually be more expected than a repeated stimulus, leading to an increase in perceived duration (Birngruber et al., 2017; Fromboluti, 
Jones, \& McAuley, 2013; Kim \& McAuley, 2013; Lin \& Shimojo, 2017; Matthews \& Gheorghiu, 2016; McAuley \& Fromboluti, 2014; Skylark \& Gheorghiu, 2017; Wehrman et al., 2018).

It is often assumed that an oddball extends the perceived duration of an event such that we retrospectively remember the event as lasting longer than it actually does. However, an alternative proposition is that an oddball is perceived to onset earlier; when a nonrepeated stimulus occurs, the 'gating' of when that stimulus starts is earlier because of increased attention drawn by the nonrepeated stimulus, or higher expectation of a nonrepeated stimulus. Because the nonrepeated stimulus is gated earlier, the perceived duration of the nonrepeated stimulus is extended. This possibility has been raised in several articles (e.g., Fromboluti et al., 2013; Lin \& Shimojo, 2017; McAuley \& Fromboluti, 2014; Wehrman et al., 2018); however, the standard oddball duration illusion, examining only perceived duration, cannot differentiate an onset-based effect.

In likely the most convincing article regarding onset-based oddball effects, Lin and Shimojo (2017) showed that simply being a target, whether the actual image was expected or not, resulted in prolonged perceived duration. This lent support to an attentional account of the oddball duration illusion, which the authors interpreted as evidence for an onset-based effect. When participants directed attention to when the target would occur, they could react more quickly to the onset of the stimulus, and this early gating led to increased perceived duration. This effect occurred irrespective of whether the stimulus was repeated or an oddball. However, the Lin and Shimojo (2017) article did not directly examine onset-based effects; it could be that directing attention in time to an event means that more information is gathered over a given amount of time, and thus the event is remembered as lasting longer irrespective of when the stimulus is perceived to onset (see Rohenkohl, Cravo, Wyart, \& Nobre, 2012; Vangkilde, Coull, \& Bundesen, 2012; Vangkilde, Petersen, \& Bundesen, 2013). To explore onset-based explanations further, in the current article the oddball duration illusion is investigated from a different angle. In two experiments, a simultaneity judgment (SJ) task is used to look for (reported) onset-based effects of oddball presentation. Two additional experiments (Experiments 3 and 4) then test whether the conditions used in Experiments 1 and 2 are able to elicit an oddball duration illusion.

\section{Simultaneity judgment tasks}

When watching an old movie or streaming content with a lag, you might notice a timing difference between the audio and the video. How close the timing of the two stimuli must be for them to be perceived as simultaneous is the essence of the SJ task. How temporally disparate two events can be and still appear as synchronous has long been of interest in psychological research (e.g., Dunlap, 1910; however, see Allan, 1975; Hirsh \& Sherrick, 1961; Sternberg \& Knoll, 1973; Welch \& Warren, 1980). Recently, the SJ task has become a popular tool in investigating perceived simultaneity (e.g., Schneider \& Bavelier, 2003; Stone et al., 2001). Specifically, this task requires participants to judge whether two stimuli occurred simultaneously when the asynchrony between them is varied. For example, in the current experiments, participants were shown one stimulus at time zero and the other between $-250 \mathrm{~ms}$ (before) and $+250 \mathrm{~ms}$ (after) the time zero stimulus. Participants then decided whether the onset of these two stimuli was simultaneous or not.

Collating the probability of choosing 'simultaneous' at each given stimulus onset asynchrony (SOA) results in an inverse $U$ curve, where the midpoint of the curve corresponds to the point of subjective simultaneity (PSS). There are several possible methods of estimating this point (see Yarrow, 2018, for a brief introduction). In the present article, an observer model was fit to the data. Specifically, the model described by García-Pérez and Alcalá-Quintana (2012) and AlcaláQuintana and García-Pérez (2013) was used, in which the two stimuli (here, a sound and image) are assumed to arrive at two different latencies modelled by shifted exponential distributions. If the difference in these latencies is sufficiently small, participants report that the stimuli occurred simultaneously. Otherwise, they choose that the stimuli arrive nonsimultaneously. From this fit, the simultaneity range (SR) was estimated as the difference between the upper and lower boundary at which the participant judged simultaneity $50 \%$ of the time. The SR gives an indication of the sensitivity to whether two stimuli are simultaneous or not. The PSS was measured as the midpoint between these decision boundaries. ${ }^{1}$

\section{Oddball-induced prior entry}

One common finding from SJ and other related tasks (i.e., temporal order judgment tasks and ternary judgment tasks) ${ }^{2}$ is the 'prior entry' effect (see Spence \& Parise, 2010; Weiß \& Scharlau, 2011; Yates \& Nicholls, 2011; Zampini, Shore, \& Spence, 2005). Prior entry describes how one stimulus is perceived earlier than another stimulus when it is attended to.

\footnotetext{
${ }^{1}$ This definition was chosen, rather than the peak of the function, as it corresponds to the PSS in the model by Yarrow (2018); functionally, the observer model of Yarrow and Alcalá-Quintana and García-Pérez (2013) are equivalent; while the Yarrow-type model estimates the upper and lower decision boundary, the Alcalá-Quintana and García-Pérez model finds a midpoint and the range of subjective simultaneity. However, analysis of the peak gave a similar result.

${ }^{2}$ Recently, the SJ task has been preferred to other tasks due to a reduced response bias (see, for example, Spence \& Parise, 2010, for an introduction to the issue, and Yarrow, Martin, Di Costa, Solomon, \& Arnold, 2016, for experimental results comparing different task types).
} 
This idea was originally proposed by Titchener (1908) and replicated by Sternberg, Knoll, and Gates (1971). Prior entry is shown by a shift in the PSS such that for two stimuli to be perceived as simultaneous the unattended stimulus must be earlier than it would have to be otherwise. For example, attending to the visual modality results in prior entry of visual stimuli compared with auditory stimuli, and so the auditory stimulus must be presented earlier than it would have to be otherwise for simultaneity to be perceived.

Because a repeated stimulus attracts less attention than a nonrepeated stimulus, a similar effect is expected when participants perform an SJ task in which one of the two stimuli is repeated versus nonrepeated. It could be argued that perhaps deviance detection is relatively late (e.g., from studies reporting mismatched negativity occurring 200-300 ms after oddball onset; see Garrido, Kilner, Stephan, \& Friston, 2009; Näätänen, Paavilainen, Rinne, \& Alho, 2007, for review). However, there is some evidence that early sensory gating (within $50 \mathrm{~ms}$ of stimulus onset) is sensitive to whether a stimulus is novel or repeated (e.g., Boutros \& Belger, 1999; Grimm \& Escera, 2012). Further, as with the oddball duration illusion, rather than sensory gating occurring 'online' it may be a post hoc effect, in which people retrospectively decide that the oddball started earlier. Thus, the 'prior entry' of a nonrepeated stimulus compared with a repeated stimulus could occur at the time the decision of simultaneity was performed rather than the actual time of stimulus onset. Given these two possibilities, it may be possible to see an effect similar to prior entry when presenting a nonrepeated stimulus in the visual modality-for example, while presenting a standard stimulus in the auditory modality. Specifically, if the oddball duration illusion is driven by an earlier gating of the stimulus, then a shift of the PSS is expected such that a nonrepeated stimulus has an earlier PSS than a repeated stimulus in comparison with a stimulus in the opposite modality.

Using an SJ task to assess perceived onset of a stimulus may appear relatively unorthodox. However, in at least one other instance, the SJ task has been used to support an onsetbased account of perceived duration. Yarrow, Whiteley, Haggard, and Rothwell (2006) assessed perceived simultaneity in a saccade task to investigate the origin of the saccade-duration illusion, in which the duration after a saccade is perceived to last longer than it would otherwise. Similar to here, they used an auditory signal to 'tag' a point in time and see if the onset of a stimulus was perceived earlier or later than that auditory cue. Using this method, they were able to show an onsetbased effect in the saccade-duration illusion. Thus, though it may seem unorthodox, the method of simultaneity judgment has been used before to establish a similar onset-based duration illusion.

\section{The current experiments}

To test the possibility of an oddball onset effect, two experiments were run using an audio-visual SJ task. In Experiment 1, each trial was initiated with either a tone (Experiment 1a) or a visual stimulus (Experiment 1b). Following this, another tone or visual stimulus, respectively, was presented, which either matched or mismatched the stimulus initiating the trial. Between $250 \mathrm{~ms}$ before $(-250 \mathrm{~ms})$ and $250 \mathrm{~ms}$ after $(+250$ $\mathrm{ms}$ ) the target stimulus, another stimulus was delivered in the nontarget modality. Participants then judged whether the two stimuli onset at the same time or not.

In Experiments $2 \mathrm{a}$ and $2 \mathrm{~b}$, the effects of target auditory (Experiment 2a) or visual (Experiment 2b) stimulus rarity were tested. In these two tasks, participants made the same judgment under the same conditions as in Experiment 1, except that there was no initiating stimulus to each trial. Rather, one tone (Experiment 2a) or visual stimulus (Experiment 2b) was presented the majority of the time, while the other was rarer. This examines the effects of rarity, rather than repetition, on the perceived onset of the target stimulus. Given that both repetition and rarity have been shown to result in an oddball duration illusion, both these methods were of interest (e.g., Ernst et al., 2017; Matthews, 2015; Mento, Tarantino, Sarlo, \& Bisiacchi, 2013).

PSS and SR, as mentioned above, were examined, as were reaction times (RTs). RTs are informative of the point of maximum uncertainty (PMU), defined as the objective duration in which participants are slowest to respond and thus most uncertain of which response to make (for further discussion and applications of the method, see Birngruber et al., 2017; Birngruber et al., 2014; Dyjas \& Ulrich, 2014).

In addition to the above experiments, two additional tasks were performed on separate groups of participants. In the third experiment, participants were required to judge the duration of a target stimulus, which was preceded by a short, obscured, initial stimulus. One possible issue in Experiment 1 is that the duration of the first stimulus was relatively short. It may be that such a brief stimulus does not induce an oddball duration illusion in the subsequent target stimulus, a possibility that has not yet been tested. Further, in Experiment 2, perhaps the brevity of the target stimuli did not result in a rare-common distinction. Thus, this third experiment tests whether a short duration stimulus can have said effect.

In the third experiment, an initial (prime) stimulus was displayed for $25 \mathrm{~ms}$. Immediately after displaying the prime, a grey-coloured block fully occluding the area of the prime (a backward mask; see, for example, Eimer, 1999; Schlaghecken, Bowman, \& Eimer, 2006; Tucker \& Ellis, 2004). Following the mask, a target stimulus was displayed for a variable duration, which participants were required to judge, similar to a classic oddball duration experiment (e.g., Matthews, 2011, 2015). 
In Experiment 4, participants performed a standard oddball duration task with slight modification. In Experiments 1 and 2, it could be possible that presenting a stimulus in the opposite modality 'broke' the illusion. Thus, on every trial in Experiment 4, a tone was played concurrently with the onset of the target stimulus. Further, in the normal oddball duration task, participants are only required to judge a subset of trials (e.g., Ernst et al., 2017), or are shown at least two trials in a row, the last of which either matches or mismatches the prior (e.g., Matthews, 2011, 2015; Wehrman et al., 2018). In Experiment 4, participants were required to judge the duration of every trial, and only the rarity of the target stimuli was changed. Along with being an interesting extension to the oddball duration literature, this experiment aims to show that rarity alone, when judging every trial and when presented with a concurrent onset marking stimulus in the opposite modality, could still produce the oddball duration illusion.

\section{Experiment 1}

The first experiment presented here tests whether repeating a target results in a shift of PSS compared with a nonrepeated stimulus.

\section{Method}

\section{Participants}

Experiments 1a and $1 \mathrm{~b}$ consisted of 20 participants each. Participant data were replaced if the participant either failed to perform the task appropriately (e.g., responding to the colour of the circle rather than simultaneity in Experiment 1b), or if a $\chi^{2}$ test revealed a poor fit between the model and data (i.e., $p<.05$ ). Using these criteria, three participants were replaced in Experiment 1a, and two participants were replaced in Experiment 1b. In Experiment 1a, the mean age of the included participants was 20.9 years $(S D=4.0$ years), no participants were left-handed (self-reported), and six participants were male. In Experiment 1b, the mean age of the included participants was 20.2 years $(S D=2.62$ years), three participants were left-handed, and five participants were male.

Participants were recruited from the Macquarie University psychology register and given one course credit for their participation, or 5 AUD. Participants provided written consent, in accordance with the Declaration of Helsinki. The experiment was approved by the Macquarie University Ethics Committee. All participants had self-reported normal or corrected-tonormal sight, normal colour vision, and normal hearing.

Participants were instructed at the beginning of each experiment not to perform rhythmical activity, such as tapping, humming, or nodding, and not to count, as this can interfere with timing processes (Grondin \& Killeen, 2009; Grondin, Ouellet, \& Roussel, 2004).

\section{Equipment}

Experimental stimuli were presented on a Samsung SyncMaster SA950 (27 inch) monitor controlled by a Dell Optiplex 9010 PC (8 GB RAM, 3.2 GHz Intel i5-3470 CPU) running 64-bit Windows 7 . Tasks took place in dimly lit, sound dampened rooms with participants seated $0.8 \mathrm{~m}$ away from their monitor. Neurobehavioral System's Presentation (Version 18.3) was used to present stimuli and record responses.

\section{Stimuli and procedure}

In both Experiments 1a and 1b, a stimulus was initially presented for $100 \mathrm{~ms}$. In Experiment 1a, this initial stimulus was a $1000 \mathrm{~Hz}$ or $500 \mathrm{~Hz}$ sinusoidal tone. In Experiment 1b, this initial stimulus was either a blue (RGB: $0,128,255)$ or grey (RGB: 113, 113, 114) circle of 125 pixel diameter. The initial tone/circle was counterbalanced between participants.

This was followed by a 1,200-ms blank screen. Note, because temporal expectancy has been shown to affect the oddball duration illusion (Wehrman et al., 2018), the timing from the onset of the trial to the target stimulus was kept the same so as to avoid any temporal expectancy-based effects. After the blank screen, another tone (Experiment 1a) or circle (Experiment 1b) was displayed for $50 \mathrm{~ms}$. This stimulus either matched the stimulus presented at the initiation of the trial (i.e., a blue circle was followed by a blue circle in Experiment $1 b$ ), or it was followed by a nonrepeat (i.e., a blue circle was followed by a grey circle in Experiment 1b).

A stimulus in the opposing modality was presented at one of 11 possible SOAs $(-250,-170,-120,-70,-35,0,35,70$, $120,170,250 \mathrm{~ms}$ ) around this target stimulus time. In Experiment 1a, this was a grey circle, and in Experiment 1b, this was a $1000 \mathrm{~Hz}$ tone. Seven hundred ms later, a question mark was presented, indicating that a response could be made. Participants responded by pressing the ' $\mathrm{S}$ ' and ' $\mathrm{D}$ ' key on a standard keyboard, indicating whether they perceived the onset of the two stimuli as simultaneous or different, respectively. A blank screen was then presented for between $600 \mathrm{~ms}$ and $800 \mathrm{~ms}$ before the next trial started (Fig. 1 shows one trial of Experiment 1b; Experiment 1a was the same except the circles and sounds were replaced with one another).

Participants performed seven blocks of 66 trials. In each block, participants were shown each SOA three times in both the repeated and nonrepeated conditions. Trials were presented in a randomized order, and between blocks participants had a self-regulated break. 


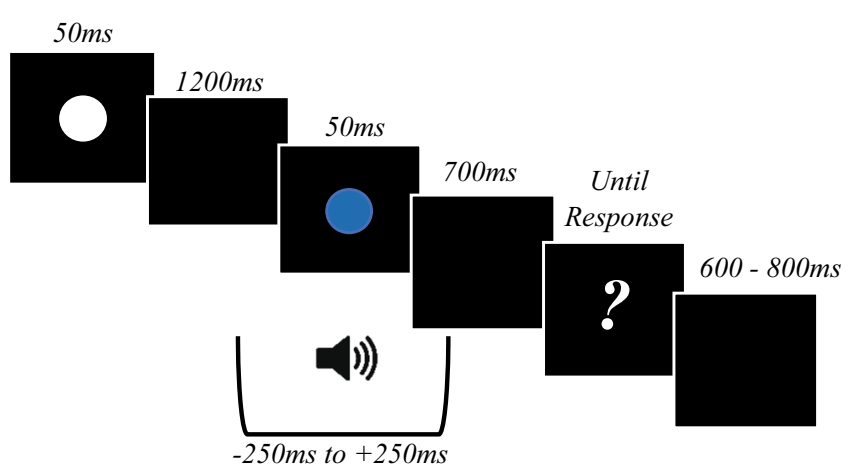

Fig. 1 General outline of the SJ task used in Experiment 1b. Shown is a nonrepeated stimulus. Experiment 1a had a similar design, except that the visual stimuli were replaced with sound stimuli and vice versa

\section{Analysis}

For both Experiments 1a and 1b, the first 20 trials were discarded as familiarization to the task. Then, for each SOA, the average percentage of 'simultaneous' decisions was calculated. Then, the function 'fit SJ2' was used, as provided in the Supplementary Material to Alcalá-Quintana and García-Pérez (2013; see their article for further details, which are beyond the scope of the current paper). In brief, this finds the bestfitting parameters using maximum-likelihood parameter estimation. Bounds were set for each parameter as described in Alcalá-Quintana and García-Pérez (2013), and visual inspection of the model fits showed adequate fitting, supported by $x^{2}$ tests with $p$ values above $.05 .^{3}$ The model was fit with error parameters estimated for the test stimulus being first, the reference stimulus being first, and simultaneous presentation. This maximally flexible model was chosen to allow these parameters to absorb deviation from the other parameters, reducing the likelihood of Type I errors. The function 'PerformanceMeasures' was then used. This function extracts the PSS, defined as the midpoint between the decision boundaries, and the SR, defined as the range between the $50 \%$ decision points.

Statistical analysis was performed using R (R Core Team, 2015). An ANOVA was run with the condition as a within-subjects factor, and whether the targe was in the auditory or visual modality as a between-subjects factor (i.e., Experiments 1a and 1b). Welch's $t$ tests were used for post hoc analysis using the Holm correction (Holm, 1979) and Cohen's $d$ are reported. Bayes factors (BF) were also calculated using the 'BayesFactor' package from R (Morey, Rouder, Jamil, \& Morey, 2015). These are interpreted in line with the Jeffreys (1961) paper. Note that $t$ tests on each experiment individually presented a similar result; we chose a between-subjects analysis, as this increased the power of the experiment overall.

\footnotetext{
${ }^{3}$ Experiment 1a: mean $\chi^{2}=4.23, p=.413$. Experiment 1b: mean $\chi^{2}=4.50, p$ $=.399$. Experiment 2a: mean $\chi^{2}=3.93, p=.474$. Experiment $2 \mathrm{~b}:$ mean $\chi^{2}=$ $4.58, p=.423$.
}

RT data were also examined. This is because response speed may be indicative of response uncertainty (see Balc1 \& Simen, 2014; Simen, Balci, Cohen, \& Holmes, 2011) and informative in judgment tasks similar to that used here (e.g., Cardoso-Leite, Gorea, \& Mamassian, 2007; Heath, 1984). However, because there was a mandatory gap between the offset of the oddball interval and the onset of the question mark allowing the response, the effect of the manipulations on RTs may be reduced. To analyze the response speed data, those trials in which participants responded slower than $4,000 \mathrm{~ms}$ were discarded as outliers. The point of maximal uncertainty (PMU) of the RT distribution across the SOAs was defined as the objective duration corresponding to the maximum of the RT distribution. The maximum was found by using waveform moment analysis ${ }^{4}$ on each condition in each experiment (Cacioppo \& Dorfman, 1987), and the PMU was then compared using a Welch's $t$ test (for further discussion and applications of the method, see Birngruber et al., 2017; Birngruber et al., 2014; Dyjas \& Ulrich, 2014). The SOA for when both the auditory and visual stimuli were simultaneous (i.e., $\mathrm{SOA}=0$ ) was changed to 1 , as otherwise these points would not contribute to the PMU. However, this made no significant difference to the results.

\section{Results}

To test for a generalized response bias, the mean percentage of times that participants responded 'synchronous' per condition was compared. This revealed that participants were not responding significantly more when the target was repeated than when it was nonrepeated, $t(39)=.319, p=.752, d=.02$. The BF of this difference was 0.18 , indicating moderate evidence for no real difference in 'simultaneous' response rates between the conditions. Figure 2 presents overall responses for Experiments 1a (left) and 1b (right) across both repeated and nonrepeated conditions.

An ANOVA was then performed on whether the target stimulus was repeated or not (within subjects), and whether the target was in the auditory or visual domain (between subjects). The mean SR was $299 \mathrm{~ms}$. The SR did not significantly vary given the experiment performed, $F(1,38)=.763, p=$ $.388, \eta_{\mathrm{p}}{ }^{2}=.02, \mathrm{BF}=.36$, or whether the stimulus was repeated or novel, $F(1,38)<.001, p=.987, \eta_{\mathrm{p}}^{2}=.00, \mathrm{BF}=.17$. The interaction between these predictors was significant, $F(1,38)$

\footnotetext{
${ }^{4}$ In brief, this involved normalizing each mean RT for each binned duration in each condition by dividing by the sum of the mean RTs for each participant. Each binned duration was then multiplied by its corresponding weight, and the results were summed within each condition for each participant.
} 


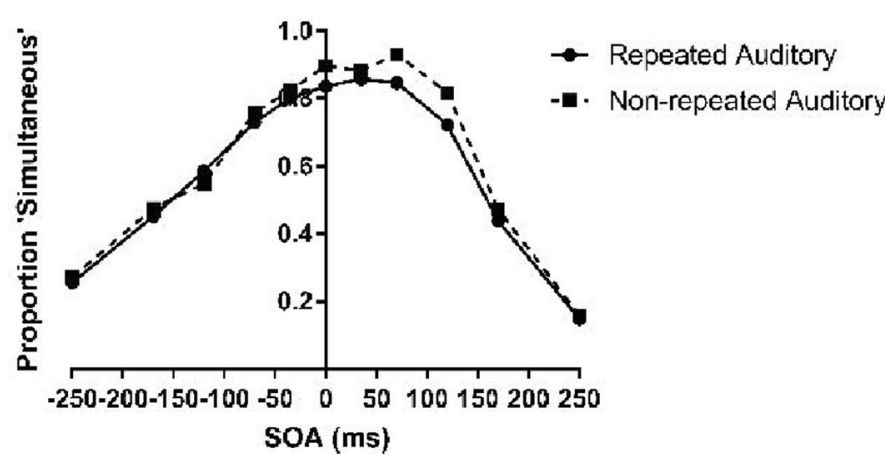

Fig. 2 Left: Mean probability of choosing 'simultaneous' across Experiment 1a. Right: Mean probability of choosing 'simultaneous' across Experiment $1 \mathrm{~b}$. Solid lines indicate that the stimulus initiating

$=5.18, p=.029, \eta_{\mathrm{p}}{ }^{2}=.12$. However, none of the comparisons were significantly different from any of the others, maximum, $t(32.3)=1.93, p=.377, d=.61$, when comparing the nonrepeated SR between the two tasks. This effect was not examined further.

The mean PSS was $-3.8 \mathrm{~ms}$. When the sound was presented as time zero (Experiment 1a), the visual stimulus had to arrive $22 \mathrm{~ms}$ earlier to be perceived as simultaneous, while if the visual stimulus was presented at time zero (Experiment 1b), the sound had to be presented $30 \mathrm{~ms}$ later than the target. This difference in PSS was significant, $F(1,38)=33.2, p<.001, \eta_{\mathrm{p}}{ }^{2}=.47, \mathrm{BF}=1.8$ $\times 10^{5}$; see Fig. 3). There was no significant effect of whether the target stimulus was repeated or novel, $F(1$, $38)=.425, p=.518, \eta_{\mathrm{p}}{ }^{2}=.01, \mathrm{BF}=.21$. The BF provided moderate support for no genuine effect of whether the target was repeated or not on PSS. There was also no interaction effect, $F(1,38)=.338, p=.565, \eta_{\mathrm{p}}{ }^{2}=.01$.

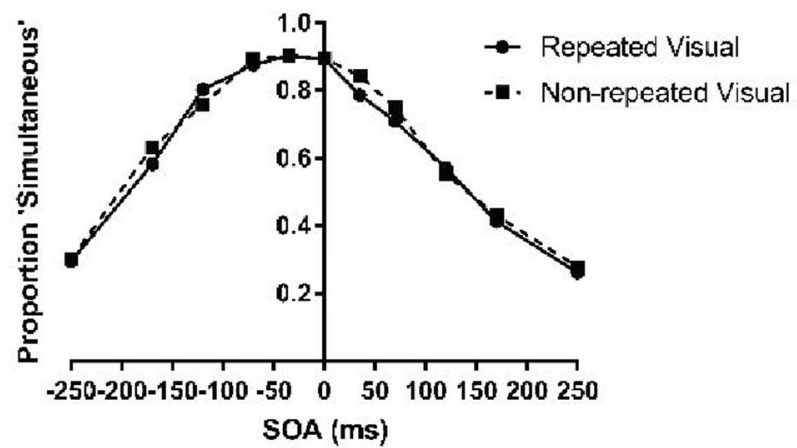

the trial and the target were the same; a broken line indicates that the stimulus initiating the trial and the target stimulus were not the same

For the RT analysis, $0.05 \%$ of trials were removed as slow responses. The PMU was not significantly affected by any factors, modality: $F(1,38)=1.05, p=.312, \eta_{\mathrm{p}}^{2}=.03, \mathrm{BF}=$ .48 ; target repetition: $F(1,38)=.187, p=.668, \eta_{\mathrm{p}}{ }^{2}=.00, \mathrm{BF}=$ .19 ; interaction: $F(1,38)=.381, p=.541, \eta_{\mathrm{p}}^{2}=.01$. The mean PMU was at $+2 \mathrm{~ms}$. This indicates that the mean point at which subjects were maximally uncertain regarding whether the two stimuli were simultaneous was near the objective point of simultaneity.

\section{Experiment 2}

In Experiment 1, there was no effect of stimulus repetition on PSS. However, while repetition of a stimulus can lead to an oddball duration effect, so can stimulus rarity (e.g., Ernst et al., 2017). Therefore, in Experiment 2, stimulus rarity rather than repetition was tested for an effect on PSS.

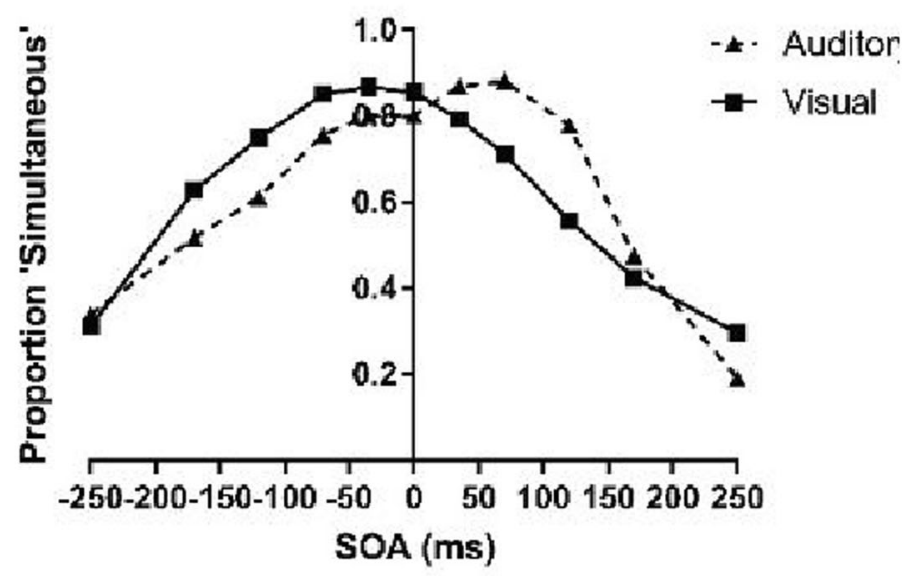

Fig. 3 Left: Mean probability across all participants of choosing that the visual and auditory stimulus occurred simultaneously in the visual (solid line) and auditory (broken line) modality experiments. Right: Difference

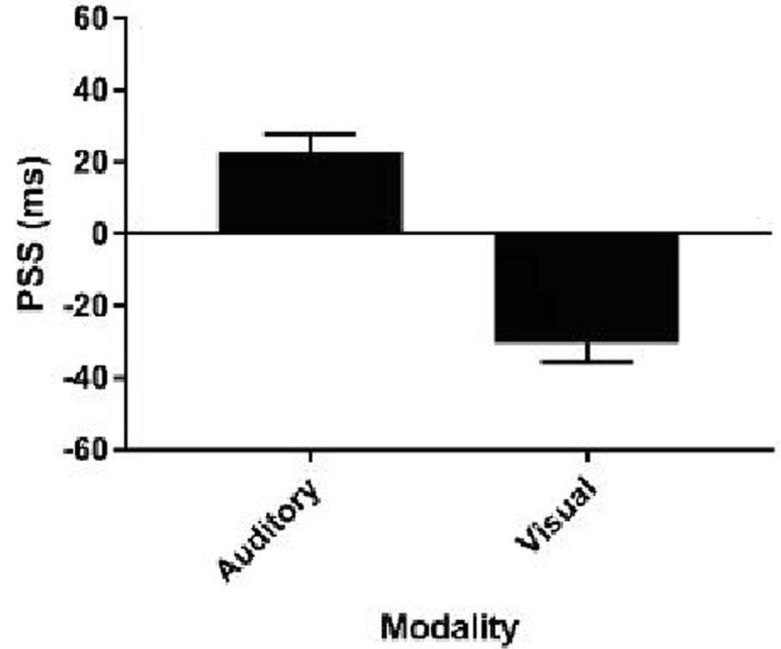

in PSS between the modalities specified as zero. Error bars represent one standard error of the mean 


\section{Method}

\section{Participants}

Four participants were replaced in Experiment 2a, and three participants were replaced in Experiment $2 b$ using the same criteria for inclusion from Experiment 1. In Experiment 2a, the mean age of included participants was 20.2 years $(S D=3.24$ years), two participants were left-handed, and nine participants were male. In Experiment $2 b$, the mean age of included participants was 18.9 years $(S D=1.07$ years), no participants were left-handed, and eight participants were male.

\section{Stimuli and procedure}

The equipment used in Experiment 2 were the same as in Experiment 1. The stimuli used were the same as Experiment 1 and were again counterbalanced.

Experiments $2 \mathrm{a}$ and $2 \mathrm{~b}$ were run in the same manner as Experiment 1, except that there was no trial initiation signal. Each trial started with a blank screen varying between $700 \mathrm{~ms}$ and $1,000 \mathrm{~ms}$, followed by a tone (Experiment $2 \mathrm{a}$ ) or a circle (Experiment $2 \mathrm{~b}$ ). The other signal (circle or tone, respectively), occurred at one of 11 possible SOAs $(-250,-170,-120$, $-70,-35,0,35,70,120,170,250 \mathrm{~ms}$ ), as per Experiment 1. There was a 500-ms gap between the target stimulus and the question mark indicating a participant could make their response. Following response, the next trial was initiated.

The main manipulation in Experiments $2 \mathrm{a}$ and $2 \mathrm{~b}$ was that the target tone (Experiment 2a) or target circle (Experiment 2b) - that is, one of the two stimuli (high or low tone, Experiment 2a, blue or grey circle, Experiment 2b), was presented $\approx 71 \%$ of the time, while the other stimulus was presented $\approx 29 \%$ of the time. Thus, the rarity of the stimulus, instead of whether it was repeated or nonrepeated, determined if the stimulus was odd or not. Participants completed 10 blocks of 77 trials, in which 22 were rare and 55 were common. Analysis was as per Experiment 1.

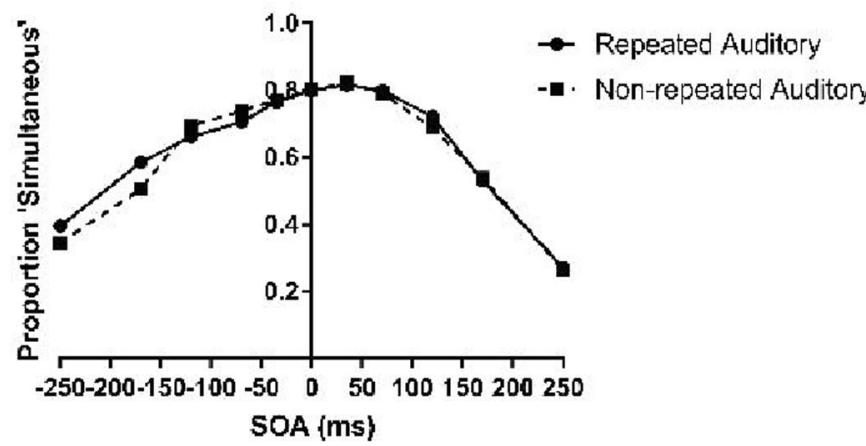

Fig. 4 Left: Mean probability of choosing 'simultaneous' across Experiment 2a. Right: Mean probability of choosing 'simultaneous' across Experiment $2 \mathrm{~b}$. Solid lines indicate that the stimulus initiating

\section{Results}

To test for a generalized response bias, the mean percentage of times that participants responded 'synchronous' per condition was again tested. This revealed that participants were not responding significantly more when the target was frequent than when it was rare, $t(39)=.158, p=.875, d=.01$. The BF for this difference was 0.17 , indicating moderate support for this null effect being genuine. Figure 4 presents overall responses for Experiments $2 \mathrm{a}$ (left) and $2 \mathrm{~b}$ (right) across both repeated and nonrepeated conditions.

The mean SR in Experiment 2 was 287 ms. The SR difference between the experiments in this case approached significance, $F(1,38)=3.52, p=.068, \eta_{\mathrm{p}}^{2}=.08, \mathrm{BF}=1.40$; however, the $\mathrm{BF}$ analysis provided no evidence in either direction. The effect of whether the stimulus was common or rare made no significant difference to $\mathrm{SR}, F(1,38)=.599, p=.444, \eta_{\mathrm{p}}{ }^{2}=$ $.02, \mathrm{BF}=.23$. The interaction between these predictors was also insignificant, $F(1,38)=1.35, p=.252, \eta_{\mathrm{p}}{ }^{2}=.03$.

The mean PSS in Experiment 2 was -16 ms. As per Experiment 1, if the target modality was auditory (Experiment 2a), then the visual signal had to be presented $15 \mathrm{~ms}$ earlier to be perceived as simultaneous. On the other hand, if the visual signal was set as the zero point (Experiment $2 b$ ), then the sound had to be presented $46 \mathrm{~ms}$ later. This difference in PSS was significant, $F(1,38)=36.0, p<.001$, $\eta_{\mathrm{p}}^{2}=.49, \mathrm{BF}=1.7 \times 10^{5}$; see Fig. 5 , left and middle. There was no significant effect of whether the target stimulus was common or rare, $F(1,38)=.221, p=.641, \eta_{\mathrm{p}}^{2}=.01, \mathrm{BF}=.27$. The $\mathrm{BF}$ indicates moderate support for no significant effect of stimulus type. There was also no interaction effect, $F(1,38)=$ $1.04, p=.313, \eta_{\mathrm{p}}^{2}=.03$.

For the RT analysis, $0.3 \%$ of trials were removed as slow responses. The PMU was significantly later in the visual condition $(+8.1 \mathrm{~ms})$ than in the sound condition $(+3.3 \mathrm{~ms}) ; F(1$, $38)=14.0, p<.001, \eta_{\mathrm{p}}^{2}=.27, \mathrm{BF}=219$; see Fig. 5 , right. Neither the rarity of the stimulus, $F(1,38)=.303, p=.585, \eta_{\mathrm{p}}{ }^{2}$ $=.01, \mathrm{BF}=.20$, nor the interaction, $F(1,38)<.001, p=.999$, $\eta_{\mathrm{p}}^{2}=.00$, reached significance.

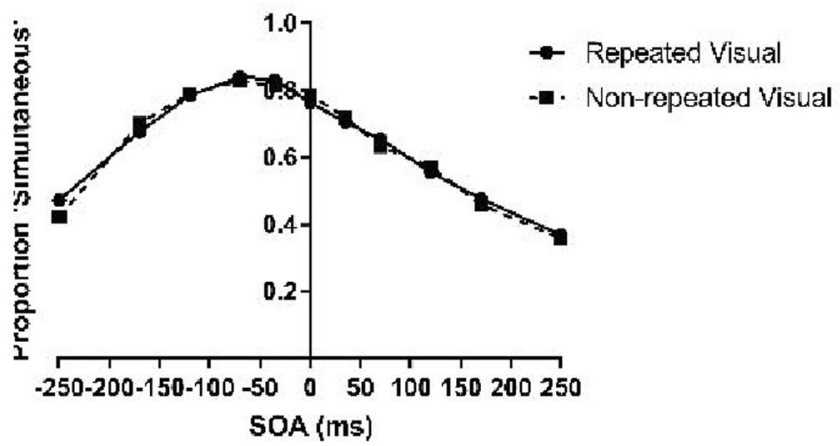

the trial and the target were the same; a broken line indicates that the stimulus initiating the trial and the target stimulus were not the same 


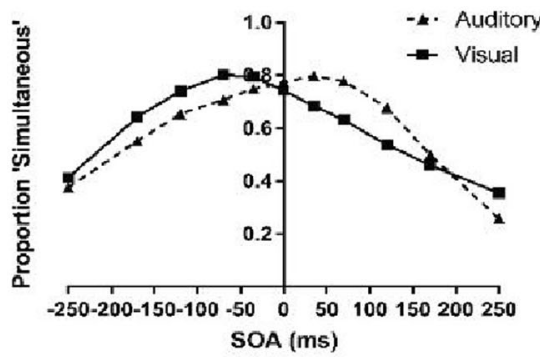

Fig. 5 Left: Mean probability across all participants of choosing that the visual and auditory stimulus occurred simultaneously in the visual (solid line) and auditory (broken line) modality tasks. Middle: Difference in PSS

Together, the findings of Experiments 1 and 2 did not provide evidence in favour of an onset-based explanation of the oddball duration illusion. Instead, Bayesian analysis provided moderate evidence against such an effect existing in the current experiments. As per prior research, findings do reconfirm that auditory stimuli are perceived to onset earlier than visual stimuli. In the next two experiments, the parameters of the oddball duration illusion are tested in order to demonstrate that the conditions presented in Experiments 1 and 2 are capable of producing an oddball duration illusion.

\section{Experiment 3}

Experiments 1 and 2 did not demonstrate a change in PSS depending on either repetition or rarity. While this is unlikely to be due to the duration of the target stimulus (as an onset-based effect would be inherently at the beginning of the stimulus being presented), perhaps specifically in Experiment 1, the duration of the trial initiator was not long enough to result in an oddball effect. Similarly, in Experiment 2, perhaps the durations of the targets were so short that there was not an effect of target rarity. Thus, in Experiment 3, a short duration, occluded, conditioning stimulus was presented to examine whether an oddball duration illusion could still be found under such conditions.

\section{Method}

\section{Participants}

Twenty people participated in Experiment 3; however, two were rejected because they failed to perform the task appropriately (i.e., PSE was outside the tested durations or $>40 \%$ of the extreme judgments were incorrect). Of the 18 remaining, the mean age was 22.2 years ( $S D=4.3$ years), with four males and one left-handed participant.

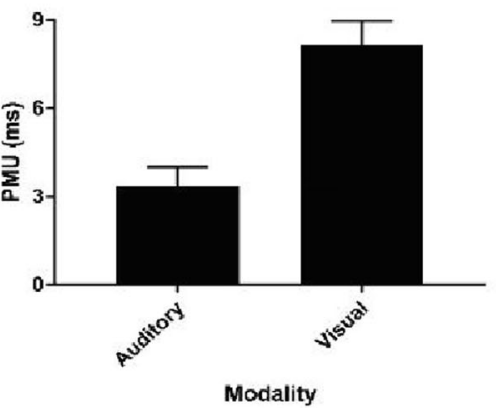

between the modalities specified as zero. Right: Difference in PMU between the task modalities. Error bars represent one standard error of the mean

\section{Stimuli and procedure}

The equipment used for this experiment were as per Experiments 1 and 2. In a 'standard familiarization' phase, participants were presented the standard duration that was used to judge whether a test interval was longer or shorter than in a subsequent section of the experiment. Though this method was used to more quickly familiarize participants to what is 'short' and 'long,' it is not strictly necessary, as even without the presentation of a standard participants can still make a judgment regarding the duration of a test interval (Wearden $\&$ Bray, 2001). The familiarization phase included 20 presentations of a filled white circle (150 pixel diameter) presented centrally on the screen. Each circle was presented for $700 \mathrm{~ms}$ (the mean duration of the test stimulus), followed by a random gap of between 400 and $600 \mathrm{~ms}$. Following the familiarization phase, participants began the testing phase of the experiment.

Each trial of the testing phase was initiated with one of four shapes: a hexagon, pentagon, triangle, or circle, presented in grey (RGB: 125, 125, 125), for $25 \mathrm{~ms}$. Each shape had a radius of 75 pixels, as controlled by the presentation software. This was followed by a solid grey square with the same radius, presented for $100 \mathrm{~ms}$, acting as a mask. Following this, one of the same four shapes was presented for a random duration (limited by the frame rate of the screen) between 300 and $1,100 \mathrm{~ms}$. Each of the four initial shapes occurred $25 \%$ of the time, while the target shape following the mask was the same as the prime $50 \%$ of time. Five hundred ms after the target disappeared, a question mark was presented, indicating that participants were able to respond. After response, a random $400 \mathrm{~ms}$ to $600 \mathrm{~ms}$ gap preceded the next trial.

Participants responded by pressing the 'L' and 'S' key on a standard keyboard, indicating whether they perceived the duration of the target to be 'longer' or 'shorter,' respectively, than the standard from the first phase of the experiment.

Participants performed eight blocks of this task, consisting of 48 trials each. Twenty-four of these trials showed a congruent prime to target paring, while the other 24 trials were 
incongruent (two of each shape pairing). In the final block, instead of judging the duration of the final shape, participants judged whether the shape prior to the mask was the same (' $\mathrm{S}$ ') or different ('L') with the shape presented after the mask. The general trial procedure is shown in Fig. 6 .

\section{Analysis}

The first five trials of each data set were discarded. Analysis was performed using R (R Core Team, 2015) and the packages 'ez' (Lawrence, 2013), and 'quickpsy' (Linares \& López-Moliner, 2016). For each participant under each condition, the psychometric function was estimated based on the proportion of times the participant indicated that the target was longer than the standard presented at the beginning of the experiment. The quickpsy program fits a cumulative normal function to the data using direct likelihood maximization by nonparametric bootstrapping of 1,000 samples (see Linares \& López-Moliner, 2016, for further information). From the psychometric function, the point of subjective equality, defined as the point where the probability of a 'longer' response was $50 \%$, was estimated. The PSEs were then used as the dependent variable in subsequent statistical analyses.

RTs were binned into five equally sized test durations of $160 \mathrm{~ms}$ each. The middle of each of these was taken as the dependent variable: $380 \mathrm{~ms}, 540 \mathrm{~ms}, 700 \mathrm{~ms}, 860 \mathrm{~ms}$, and $1,020 \mathrm{~ms}$. The PMU was then calculated as above.

Finally, the Weber ratio (WR) for each condition and participant was calculated as the difference in the duration on the psychometric curve at which participants chose 'long' $75 \%$ and $25 \%$ of the time, divided by two and normalised by the bisection point. The WR was then run through the same statistical analysis as performed on the PSE. This measure was used to quantify the change discriminability under each condition.

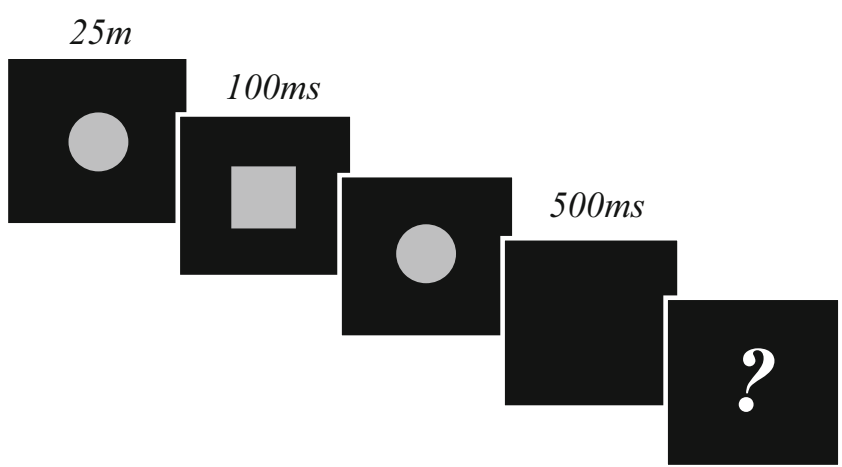

Fig. 6 General outline of a single trial of the masked priming experiment. Shown is a congruent trial in which the shape after the mask matches the shape prior to the mask. Each trial was initiated with a prime shape for 25 $\mathrm{ms}$, then a $100-\mathrm{ms}$ blank mask. The target either matched or mismatched the prime and was presented for between $300 \mathrm{~ms}$ and 1,100 ms. The question mark appeared until a participant responded. This was followed by a $400-600$-ms blank screen prior to the initiation of another trial

\section{Results}

A Welch's paired $t$ test showed that the PSE was significantly later if the prime and the target were congruent (PSE $=730$ $\mathrm{ms}$ ) rather than incongruent (PSE $=708 \mathrm{~ms}), t(17)=2.53, p=$ $.022, d=.60$. This indicated that if the prime and the target were congruent, then the target was reported to last for a shorter amount of time (see Fig. 7).

Participants performed significantly better than chance at guessing whether the target image matched the prime image, $t(17)=2.93, p=.009, d=.69$, averaging $60.0 \%$ correct. $^{5}$ This was not due to a response bias; the mean proportion of 'matching' responses was not significantly different than 0.5 $($ mean $=0.50), t(17)=.119, p=.907, d=.03$. Further, the proportion correct in the final task ranged between $37.5 \%$ and $87.5 \%$ correct; however, the performance on this did not correlate significantly with the PSE difference between whether the target stimulus matched or did not match with the premask stimulus $\left(r^{2}=.089, p=.228\right)$.

The average PMU was close to the objective duration of the standard $(694 \mathrm{~ms})$. This was not significantly affected by the prime congruency, $t(17)=.279, p=.784, d=.07$. This indicates no significant effect of prime congruency on the duration at which participants were most uncertain regarding whether the target duration was shorter or longer than the standard. Further, the mean WR was 0.27 , and this was also not significantly affected by prime congruency, $t(17)=.649, p=.525, d$ $=.15$.

These findings indicate that a nonrepeated stimulus is perceived to last for a longer duration than a repeated stimulus, even when the initial image is presented for a very short duration and is occluded after presentation. Presenting an initial stimulus that is difficult to perceive is an interesting extension of the oddball duration illusion generally, indicating that even such a stimulus can produce the standard perceived duration illusion. Further, the findings of Experiment 3 also demonstrate that the duration of the stimuli in Experiments 1 and 2 should have been sufficient to elicit an oddball duration illusion if present.

\section{Experiment 4}

While Experiment 3 demonstrated that short stimuli can still lead to an oddball duration illusion, other aspects of Experiments 1 and 2 may have mitigated an effect. For example, perhaps the presence of a sound detracted from the oddball duration illusion. Alternatively, because in the normal oddball duration illusion not every stimulus is judged, perhaps judgments occurring on every trial in the SJ tasks also mitigated the illusion. Therefore, in Experiment 4, participants

\footnotetext{
${ }^{5}$ This was evenly spread amongst the possible shapes.
} 


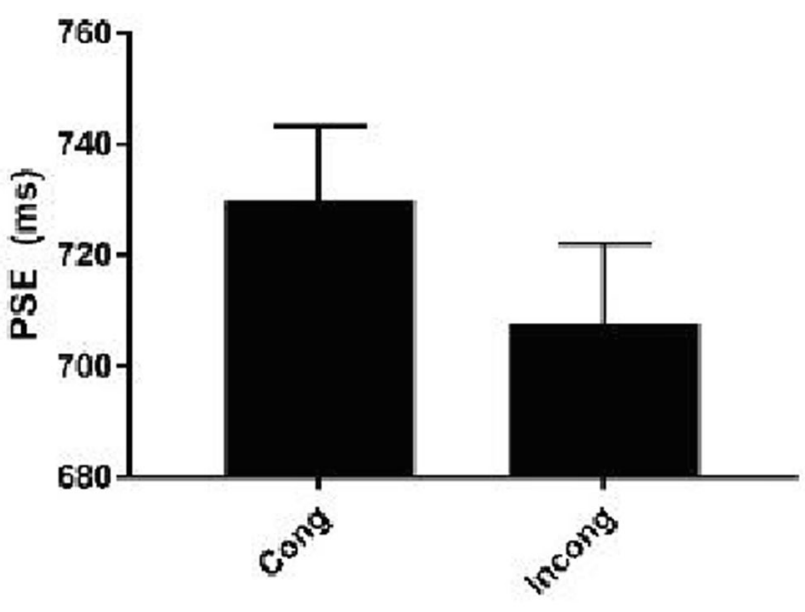

Target Stimulus

Fig. 7 Effect of prime congruency with target on perceived duration, PSE. Error bars represent one SEM

were required to judge the duration of every trial, and only the rarity of the target stimulus changed. Further, at the onset of the stimulus, a sound was played. If either of these factors explain why there is no PSS in Experiments 1 and 2, then there should also be no oddball duration illusion in the current experiment.

\section{Method}

\section{Participants}

Twenty participants took part in this study; however, four were rejected because they failed to perform the task appropriately (i.e., PSE was outside the tested durations or $>40 \%$ of the extreme judgments were incorrect). Of the 16 remaining, the mean age was 22.5 years ( $S D=6.1$ years), with four males and no left-handed participants.

\section{Equipment}

Experimental stimuli were presented on a AOC G2770PF (27 inch) monitor controlled by a Dell Optiplex 9010 PC (8 GB RAM, 3.2 GHz Intel i5-3470 CPU) running 64-bit Windows 7. Neurobehavioral System's Presentation (Version 20.2) was used to present stimuli and record responses.

\section{Stimuli and procedure}

The familiarization phase, serving the same purpose as in Experiment 3, included 20 presentations of a filled grey circle (150 pixel diameter) presented centrally on the screen. Each circle was presented for $500 \mathrm{~ms}$ (the mean duration of the test stimulus), followed by a gap of $500 \mathrm{~ms}$. Following the familiarization phase, participants began the testing phase of the experiment.

Each test trial consisted of a circle presented for between $250 \mathrm{~ms}$ and $750 \mathrm{~ms}$ (at intervals 250, 320, 380, 430, 465, 500, $535,570,620,680$, and $750 \mathrm{~ms}$ ). This circle could be coloured either grey or blue, as per Experiments $1 \mathrm{~b}$ and $2 \mathrm{~b}$. One of these colours was presented on a majority ( $\approx 71 \%$ of trials) of trials, while the other was rarer $(\approx 29 \%$ of trials). Which colour was presented the majority of times was counterbalanced between participants. Concurrent with the onset of the test circle, participants were played a $50 \mathrm{~ms}, 1000 \mathrm{~Hz}$ tone (as per Experiments 1 and 2). This tone did not serve a functional role, but was provided to ensure compatibility with the first two experiments.

After the offset of the circle, a 100-ms blank screen preceded the presentation of a question mark. This indicated the participant could respond. After response, a random 400-ms to 600-ms gap preceded the next trial. Participants responded by pressing the 'L' and 'S' key on a standard keyboard, indicating whether they perceived the duration of the target to be 'longer' or 'shorter,' respectively, than the standard from the first phase of the experiment. The general trial procedure is shown in Fig. 8 below.

Participants performed seven blocks of 77 trials each, 55 of which presented the majority colour and 22 presented the rare colour. Each target duration was presented an equal number of times per block. Trial order was randomized.

Analysis of this data was as per Experiment 3, with the exception of the calculation of the PMU. Here, the binned durations used for PMU analysis were as per the 11 possible test durations of the target stimulus.

\section{Results}

A one-sided paired $t$ test revealed that the PSE was significantly higher following a common (478 ms) compared with a rare (453 ms) stimulus, $t(15)=1.95, p=.035, d=.49$. The higher PSE following a common rather than rare stimulus shows that a common stimulus was perceived to last a shorter duration compared with a rare stimulus (see Fig. 9).

The average PMU was close to the objective duration of the standard (490 ms). This was not significantly affected by the

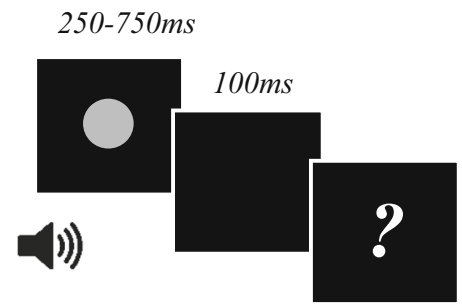

Fig. 8 General outline of a single trial Experiment 4. The question mark appeared until a participant responded. This was followed by a $400-600$ ms blank screen prior to the initiation of another trial 


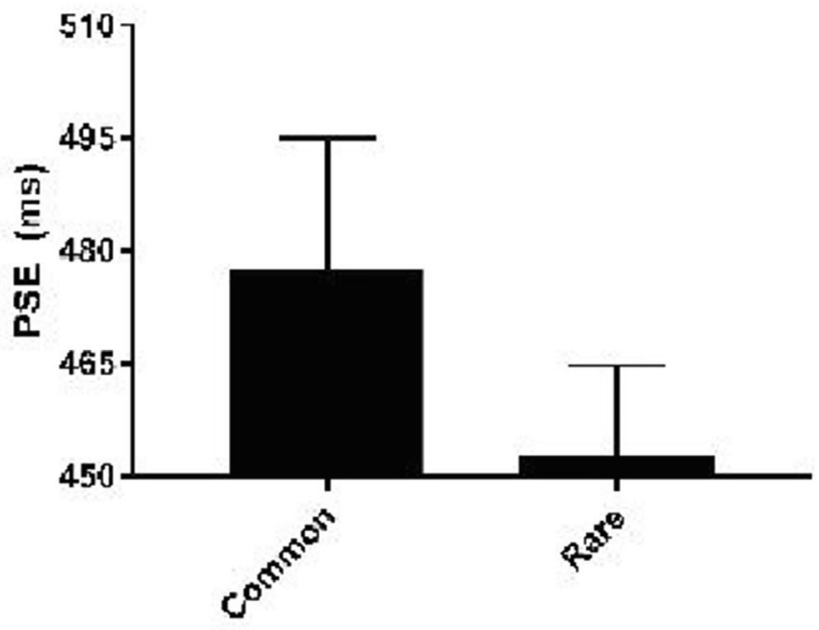

Target Stimulus

Fig. 9 Effect of target rarity on perceived duration, PSE. Error bars represent one SEM

target rarity, $t(15)=1.36, p=.194, d=.34$. This indicates no significant effect of target rarity on the duration at which participants were most uncertain regarding whether the target duration was shorter or longer than the standard. Further, the mean WR was 0.27 , and this was also not significantly affected by target rarity, $t(15)=1.08, p=.297, d=.27$.

The findings of Experiment 4 demonstrate three features of the oddball duration illusion. Firstly, it shows that presenting a concurrent sound stimulus does not eliminate the oddball duration illusion in the visual modality. Cross-modal interference could have been a concern for Experiments 1 and 2, and thus this was an important demonstration. Secondly, in the standard oddball duration experiment, only a subset of stimuli require judgment. In the current experiment, every stimulus was judged, as per in Experiments 1 and 2. Again, the findings here show that this does not eliminate the duration illusion. Finally, in the current experiment, only the rarity of the target was varied, unlike many renditions of the oddball duration illusion. Finding an oddball duration illusion here demonstrates that an oddball duration illusion should have been present specifically in Experiment 2.

\section{General discussion}

A replicable finding in the time perception literature is that a nonrepeated or rare stimulus is perceived to last longer than a repeated/common stimulus (e.g., Birngruber et al., 2017; Birngruber et al., 2014; Matthews, 2011, 2015; Wehrman et al., 2018). However, one question in this literature is whether the temporal illusion is due to an extension of perceived duration or an earlier gating of the stimulus. This is an intractable question using the common methods of the oddball duration illusion. Therefore, in two experiments, the effects of either repetition (Experiment 1) or rarity (Experiment 2 ) on simultaneity judgment was tested. Further, in Experiment 3, whether an occluded, short duration $(25 \mathrm{~ms})$ initial stimulus could induce the oddball duration illusion was also tested. In Experiment 4, participants were required to judge the duration of every stimulus. These stimuli were shown concurrently with a tone and varied as to whether they were common or rare. Though Experiments 3 and 4 may be of general interest, their main purpose was to test whether the conditions presented in Experiments 1 and 2 could still induce the oddball duration illusion.

The findings of Experiments 1 and 2 were inconsistent with the idea that the oddball duration illusion is a result of an early gating effect. Rather than finding evidence for a shift of PSS induced by the target stimuli, BF analysis showed moderate support for no such difference existing. Specifically, though the common finding that auditory stimuli are reported to onset earlier than visual stimuli was replicated (e.g., Allison, Matsumiya, Goff, \& Goff, 1977; King, 2005; Spence \& Squire, 2003), no shift was detected in relation to whether the target stimulus was a repeated stimulus (Experiment 1) or a rare stimulus (Experiment 2). It could be argued that prior entry, the mechanism underlying the attentional effects on perceived simultaneity, may be somewhat difficult to find, especially using the SJ task. However, in a review article, Spence and Parise (2010) found that prior entry can still be found using SJ tasks, though the effect is larger using temporal order judgment. Further, Yarrow et al. (2006) used a similar technique to the current article to establish prior entry-type effects in perisaccadic time dilation.

It is important to note that the conditions used in both Experiments 1 and 2 have led to an oddball duration illusion in other articles. For example, in Experiment 1, the target stimulus was either grey or blue, and thus perhaps the 'oddball' was not so odd. However, Wehrman et al. (2018) also used only two possible circle colors and still found an oddball duration effect. Further, in Experiment 2, participants did not know whether they were going to be exposed to a common or rare stimulus prior to the presentation of the target. This could be proposed to reduce the salience of the target. However, Ernst et al. (2017) found an oddball duration effect when participants were exposed to a series of colored words and were unaware of whether they would judge the duration of any given repeated or nonrepeated colored word.

One issue that could arise for the current experiments, but which has not yet been dealt with in prior research, is the duration of the stimuli used. The duration of the target stimulus was not of consequence here; an onset-based effect should be able to be found if it exists. In fact, if the target stimulus had too long a duration, an increase in overlap with the concurrent nontarget stimulus could lead to inaccurate simultaneity judgments (see Yarrow, 2018). However, it could be the case that the standard stimulus was not long enough to induce the 
oddball duration illusion. For example, in Wehrman et al. (2018), the standard was presented for $500 \mathrm{~ms}$, and in Skylark and Gheorghiu (2017) the standard was presented for $600 \mathrm{~ms}$. Perhaps there is a minimal amount of time that the standard must be presented in order for novelty to be detected. Experiment 3 tested for this possibility.

In Experiment 3, participants were required to perform a fairly standard rendition of the oddball-duration task; however, rather than a conditioning stimulus with extended duration, a 25-ms stimulus was used. Further, a mask was applied for $100 \mathrm{~ms}$ after the presentation of the conditioning stimulus. Despite these two manipulations, there was still a notable oddball duration effect of $\approx 22 \mathrm{~ms}$. It is worth noting that the prime was not successfully masked by the backward mask applied; participants were able to correctly guess the identity of the prime $60 \%$ of the time. However, there was no significant correlation between the participant successfully seeing the prime on a majority of trials and the difference in PSE between a congruent and incongruent prime-target relationship. Further, even if the backwards mask was generally ineffective, it appeared that participants were not able to guess the identity of the prime with $100 \%$ accuracy, and thus it seems that the mask occluded identification of the prime at least part of the time.

This finding demonstrates that a short duration, occluded, priming stimulus can still induce an oddball duration illusion. Thus, the duration of the standard does not appear to be an adequate explanation for the null results in the current article. Further, this finding is interesting in its own right. Most research into the oddball duration illusion requires participants to compare the duration of the standard (i.e., prime) and the target stimulus (e.g., Skylark \& Gheorghiu, 2017; Tse et al., 2004; Wehrman et al., 2018). Using such a comparison method makes the standard inherently important, and thus the duration of the standard may play a role in the oddball duration illusion. Eagleman (2008) and Pariyadath and Eagleman (2007), for example, suggested that there was not actually an extension of the perceived duration of an oddball, but rather a contraction of the perceived duration of a repeated standard. However, the method used in Experiment 3 shows that an oddball duration illusion still results from a prime that is not intrinsic to the task. This indicates that the oddball duration, and not the standard duration, is affected by the oddball duration illusion. Further, the standard does not necessarily have to be involved in the timing judgment task nor of an extended duration for the oddball duration illusion to emerge.

Experiment 4 showed that stimulus rarity alone, and when judging every trial, can still lead to an oddball duration illusion. These were important considerations given the methodologies of the first two experiments. Further, Experiment 4 demonstrated that the concurrent presentation of a sound did not break the oddball duration illusion. In fact, this adds some support to the findings of Experiments 1 and 2; if the oddball duration illusion arose because of an earlier perceived onset of an oddball stimulus, then the sound presented in Experiment 4 could have served to 'tag' the onset in both conditions and may have eliminated the oddball duration illusion. However, this did not appear to be the case; a sound presented concurrently with the onset of the target did not seem to eliminate the illusion. While this was not the point of Experiment 4, it is consistent with the findings of Experiments 1 and 2 in supporting a nononset-based effect of an oddball on perceived duration.

However, it is worth noting that the size of the oddball duration illusion was somewhat reduced in Experiment 4 compared with what might be expected given previous research (e.g., Matthews, 2011, 2015). This could have been caused by the concurrent presentation of an onset tone; however, two other explanations seem more likely. Firstly, stimulus brightness is known to affect perceived duration (Matthews, Stewart, \& Wearden, 2011; Stevens, 1966). In half of the participants of Experiment 4, the rare stimulus was grey while the common stimulus was blue. The difference in brightness of these stimuli may have served to reduce the effect of rarity in this half of participants. However, in Experiments 1 and 2, many participants took part, and, further, half of the participants were given soundbased oddball stimuli in which brightness does not play a role. Therefore, if stimulus brightness reduced the oddball duration illusion, and this resulted in an absence of the effect in the SJ tasks, then there should have been an interaction showing that the PSS was shifted specifically in the auditory, and not visual, modality. There was not support for such an effect in the results of Experiments 1 and 2.

Secondly, in Experiment 4, the familiarization phase consisted of the same grey circles used in the main test. This was not varied depending on which stimulus was rare later on in the experiment. Specifically in the case where the grey circle was shown as the rare stimulus, this initial exposure to the grey circles may have resulted in a subjectively 'less rare' experience of these circles. This in turn may have reduced the size of the oddball duration illusion. Despite these possible issues with Experiment 4, the oddball duration illusion was still present and supports the validity of the methods used in Experiments 1 and 2.

Though in the current article there was no behavioral evidence of an early gating effect in the oddball duration illusion, the use of an SJ task may be of interest in other duration illusions. Specifically, if a stimulus property is thought to elicit greater attention, perhaps a prior entry type effect might be found. It is worth noting that other investigations using stimuli that typically induce arousal, such as angry faces, do not induce prior entry (Schettino, Loeys, \& Pourtois, 2013; Schofield, Youssef, \& Denson, 2017). These findings were not related to the perceived expansion of duration of such stimuli (Fayolle \& Droit-Volet, 2014; Gil \& Droit-Volet, 2011); however, similar to the logic present here, this could 
be evidence that arousing stimuli (at least oddballs and angry faces) do not induce an onset-based illusion resulting in expanded perceived duration. Despite this, because Yarrow et al. (2006) found an onset-based effect in a known manipulation of perceived duration, it is worth further examining the role of stimulus onset on perceived duration via SJ-type tasks. The question is, which factors in a duration illusion result in onset effects?

As another use of SJ taks in duration perception research, it is known that both implicitly and explicitly cued stimuli appear to last longer than uncued or falsly cued stimuli (Bausenhart, Rolke, \& Ulrich, 2008; Birngruber et al., 2017; Grondin \& Rammsayer, 2003; Seifried \& Ulrich, 2011; Ulrich et al., 2006; Wehrman et al., 2018). Specifically, Seifried and Ulrich (2011) found that exogenous cuing of stimulus location can increase the perceived duration of that stimulus, a manipulation that has also been shown to shift PSS (e.g., Kanai, Ikeda, \& Tayama, 2007; Shore, Spence, \& Klein, 2001; Yates \& Nicholls, 2009). Relatedly, directing attention temporally also tends to increase perceived duration (Grondin \& Rammsayer, 2003; McAuley \& Fromboluti, 2014; Wehrman et al., 2018). However, directing attention temporally only appears to improve simultaneity judgment accuracy (i.e., SR) without shifting the PSS (Bausenhart et al., 2008; Correa, Sanabria, Spence, Tudela, \& Lupiáñez, 2006). It is interesting to note that temporal attention does not affect the PSS, while spatial attention does. The relationship between these types of attention, perceived duration, and the perceived onset of stimuli deserves further research.

\section{Conclusion}

In the current article, the SJ task did not provide evidence that the oddball duration illusion is caused by early gating. Instead, there was moderate support from BFs to support no such effect existing. Up to this point, the early gating hypothesis has been difficult to assess due to the nature of the oddball duration illusion experiment; however, the use of the SJ task is a novel approach to such problems and may be useful in future investigations relating to the locus of effect of duration illusions. Finally, as an auxiliary finding, Experiment 3 provided evidence that the duration of the standard stimulus is not a primary driver of the oddball duration illusion. Further, Experiment 4 demonstrates that the oddball duration illusion can emerge even when participants are presented with a simultaneous standard sound at the onset of the target, and when only the rarity of the target is manipulated.

Open practices statement None of the experiments was preregistered. Data will be made available upon request.

\section{References}

Alcalá-Quintana, R., \& García-Pérez, M. A. (2013). Fitting model-based psychometric functions to simultaneity and temporal-order judgment data: MATLAB and R routines. Behavior Research Methods, 45(4), 972-998.

Allan, L. G. (1975). Temporal order psychometric functions based on confidence-rating data. Perception \& Psychophysics, 18(5), 369 372.

Allison, T., Matsumiya, Y., Goff, G., \& Goff, W. (1977). The scalp topography of human visual evoked potentials. Electroencephalography and Clinical Neurophysiology, 42(2), 185-197.

Balcı, F., \& Simen, P. (2014). Decision processes in temporal discrimination. Acta Psychologica, 149, 157-168. https://doi.org/10.1016/j. actpsy.2014.03.005

Bausenhart, K., Rolke, B., \& Ulrich, R. (2008). Temporal preparation improves temporal resolution: Evidence from constant foreperiods. Perception \& Psychophysics, 70(8), 1504-1514. https://doi.org/10. 3758/PP.70.8.1504

Birngruber, T., Schröter, H., Schütt, E., \& Ulrich, R. (2017). Stimulus expectation prolongs rather than shortens perceived duration: Evidence from self-generated expectations. Journal of Experimental Psychology: Human Perception and Performance, 44(1), 117-127. https://doi.org/10.1037/xhp0000433

Birngruber, T., Schröter, H., \& Ulrich, R. (2014). Duration perception of visual and auditory oddball stimuli: Does judgment task modulate the temporal oddball effect? Attention, Perception, \& Psychophysics, 76(3), 814-828. https://doi.org/10.3758/s13414013-0602-2

Boutros, N. N., \& Belger, A. (1999). Midlatency evoked potentials attenuation and augmentation reflect different aspects of sensory gating. Biological Psychiatry, 45(7), 917-922. https://doi.org/10.1016/ S0006-3223(98)00253-4

Cacioppo, J. T., \& Dorfman, D. D. (1987). Waveform moment analysis in psychophysiological research. Psychological Bulletin, 102(3), 421.

Cai, M. B., Eagleman, D. M., \& Ma, W. J. (2015). Perceived duration is reduced by repetition but not by high-level expectation. Journal of Vision, 15(13), 19-19. https://doi.org/10.1167/15.13.19

Cardoso-Leite, P., Gorea, A., \& Mamassian, P. (2007). Temporal order judgment and simple reaction times: Evidence for a common processing system. Journal of Vision, 7(6), 11-11.

Dunlap, K. (1910). The complication experiment and related phenomena. Psychological Review, 17(3), 157.

Dyjas, O., \& Ulrich, R. (2014). Effects of stimulus order on discrimination processes in comparative and equality judgements: Data and models. The Quarterly Journal of Experimental Psychology, 67(6), $1121-1150$.

Eagleman, D. (2008). Human time perception and its illusions. Current Opinions in Neurobiology, 18(2), 131-136. https://doi.org/10.1016/ j.conb.2008.06.002

Eagleman, D., \& Pariyadath, V. (2009). Is subjective duration a signature of coding efficiency? Philosophical Transactions of the Royal Society of London B: Biological Sciences, 364(1525), 1841-1851. https://doi.org/10.1098/rstb.2009.0026

Eimer, M. (1999). Facilitatory and inhibitory effects of masked prime stimuli on motor activation and behavioural performance. Acta Psychologica, 101(2/3), 293-313.

Ernst, B., Reichard, S. M., Riepl, R. F., Steinhauser, R., Zimmermann, S. F., \& Steinhauser, M. (2017). The P3 and the subjective experience of time. Neuropsychologia, 103, 12-19.

Fayolle, S. L., \& Droit-Volet, S. (2014). Time perception and dynamics of facial expressions of emotions. PLOS ONE, 9(5), e97944. https:// doi.org/10.1371/journal.pone.0097944

Fromboluti, E. K., Jones, K. B., \& McAuley, J. D. (2013). Temporal preparation contributes to the overestimation of duration of 
'oddball' events. Frontiers in Human Neuroscience Conference Absract: 14th Rhythm Production and Perception Workshop Birmingham. https://doi.org/10.3389/conf.fnhum.2013.214.00013

García-Pérez, M. A., \& Alcalá-Quintana, R. (2012). On the discrepant results in synchrony judgment and temporal-order judgment tasks: A quantitative model. Psychonomic Bulletin \& Review, 19(5), 820 846.

Garrido, M. I., Kilner, J. M., Stephan, K. E., \& Friston, K. J. (2009). The mismatch negativity: A review of underlying mechanisms. Clinical Neurophysiology, 120(3), 453-463.

Gil, S., \& Droit-Volet, S. (2011). "Time flies in the presence of angry faces" ... depending on the temporal task used! Acta Psychologica, 136(3), 354-362. https://doi.org/10.1016/j.actpsy.2010.12.010

Grimm, S., \& Escera, C. (2012). Auditory deviance detection revisited: Evidence for a hierarchical novelty system. International Journal of Psychophysiology, 85(1), 88-92. https://doi.org/10.1016/j.ijpsycho. 2011.05.012

Grondin, S., \& Killeen, P. R. (2009). Effects of singing and counting during successive interval productions. NeuroQuantology, 7(1). https://doi.org/10.14704/nq.2009.7.1.209

Grondin, S., Ouellet, B., \& Roussel, M.-E. (2004). Benefits and limits of explicit counting for discriminating temporal intervals. Canadian Journal of Experimental Psychology/Revue canadienne de psychologie expérimentale, 58(1), 1. https://doi.org/10.1037/ h0087436

Grondin, S., \& Rammsayer, T. (2003). Variable foreperiods and temporal discrimination. The Quarterly Journal of Experimental Psychology A, 56(4), 731-765. https://doi.org/10.1080/02724980244000611

Heath, R. A. (1984). Response time and temporal order judgement in vision. Australian Journal of Psychology, 36(1), 21-34.

Hirsh, I. J., \& Sherrick, C. E., Jr. (1961). Perceived order in different sense modalities. Journal of Experimental Psychology, 62(5), 423.

Jeffreys, H. (1961). Theory of probability (3rd ed.). Oxford, England: Oxford University Press.

Kanai, K., Ikeda, K., \& Tayama, T. (2007). The effect of exogenous spatial attention on auditory information processing. Psychological Research, 71(4), 418-426.

Kim, E., \& McAuley, J. D. (2013). Effects of pitch distance and likelihood on the perceived duration of deviant auditory events. Attention, Perception, \& Psychophysics, 75(7), 1547-1558. https://doi.org/10. 3758/s13414-013-0490-5

King, A. J. (2005). Multisensory integration: Strategies for synchronization. Current Biology, 15(9), R339-R341.

Lin, Y.-J., \& Shimojo, S. (2017). Triple dissociation of duration perception regulating mechanisms: Top-down attention is inherent. PLOS ONE, 12(8), e0182639. https://doi.org/10.1371/journal.pone. 0182639

Linares, D., \& López-Moliner, J. (2016). quickpsy: An R package to fit psychometric functions for multiple groups. The R Journal, 8(1), 122-131.

Matthews, W. J. (2011). Stimulus repetition and the perception of time: The effects of prior exposure on temporal discrimination, judgment, and production. PLOS ONE, 6(5), e19815. https://doi.org/10.1371/ journal.pone.0019815

Matthews, W. J. (2015). Time perception: The surprising effects of surprising stimuli. Journal of Experimental Psychology: General, 144(1), 172-197. https://doi.org/10.1037/xge0000041

Matthews, W. J., \& Gheorghiu, A. I. (2016). Repetition, expectation, and the perception of time. Current Opinions in Behavioral Sciences, 8 , 110-116. https://doi.org/10.1016/j.cobeha.2016.02.019

Matthews, W. J., Stewart, N., \& Wearden, J. H. (2011). Stimulus intensity and the perception of duration. Journal of Experimental Psychology: Human Perception and Performance, 37(1), 303.

McAuley, J. D., \& Fromboluti, E. K. (2014). Attentional entrainment and perceived event duration. Philosophical Transactions of the Royal
Society of London B: Biological Sciences, 369(1658), 20130401. https://doi.org/10.1098/rstb.2013.0401

Morey, R. D., Rouder, J. N., Jamil, T., \& Morey, M. R. D. (2015). Package 'BayesFactor' (R Package Version 0912-2) [Computer software]. Retrieved from https://cran.r-project.org/

Näätänen, R., Paavilainen, P., Rinne, T., \& Alho, K. (2007). The mismatch negativity (MMN) in basic research of central auditory processing: A review. Clinical Neurophysiology, 118(12), 2544-2590.

New, J. J., \& Scholl, B. J. (2009). Subjective time dilation: Spatially local, object-based, or a global visual experience? Journal of Vision, 9(2), 4-4. https://doi.org/10.1167/9.2.4

Pariyadath, V., \& Eagleman, D. (2007). The effect of predictability on subjective duration. PLOS ONE, 2(11), e1264. https://doi.org/10. 1371/journal.pone.0001264

Rohenkohl, G., Cravo, A. M., Wyart, V., \& Nobre, A. C. (2012), Temporal expectation improves the quality of sensory information. Journal of Neuroscience, 32(24), 8424-8428.

Schettino, A., Loeys, T., \& Pourtois, G. (2013). No prior entry for threatrelated faces: Evidence from temporal order judgments. PLOS ONE, 8(4), e62296.

Schindel, R., Rowlands, J., \& Arnold, D. H. (2011). The oddball effect: Perceived duration and predictive coding. Journal of Vision, 11(2), 17-17. https://doi.org/10.1167/11.2.17

Schlaghecken, F., Bowman, H., \& Eimer, M. (2006). Dissociating local and global levels of perceptuo-motor control in masked priming. Journal of Experimental Psychology and Human Perceptual Performance, 32, 618-632.

Schofield, T. P., Youssef, H., \& Denson, T. F. (2017). No experimental evidence for visual prior entry of angry faces, even when feeling afraid. Emotion, 17(1), 78.

Seifried, T., \& Ulrich, R. (2011). Exogenous visual attention prolongs perceived duration. Attention, Perception, \& Psychophysics, 73(1), 68-85. https://doi.org/10.3758/s13414-010-0005-6

Shore, D. I., Spence, C., \& Klein, R. M. (2001). Visual prior entry. Psychological Science, 12(3), 205-212.

Simchy-Gross, R., \& Margulis, E. H. (2017). Expectation, information processing, and subjective duration. Attention, Perception, \& Psychophysics, 1-17. https://doi.org/10.3758/s13414-017-1432-4

Simen, P., Balci, F., Cohen, J. D., \& Holmes, P. (2011). A model of interval timing by neural integration. Journal of Neuroscience, 31(25), 9238-9253.

Skylark, W., \& Gheorghiu, A. (2017). Further evidence that the effects of repetition on subjective time depend on repetition probability. Frontiers in Psychology, 8, 1915. https://doi.org/10.3389/fpsyg. 2017.01915

Spence, C., \& Parise, C. (2010). Prior-entry: A review. Consciousness and Cognition, 19(1), 364-379. https://doi.org/10.1016/j.concog. 2009.12 .001

Spence, C., \& Squire, S. (2003). Multisensory integration: Maintaining the perception of synchrony. Current Biology, 13(13), R519-R521.

Sternberg, S., \& Knoll, R. L. (1973). The perception of temporal order: Fundamental issues and a general model. In S. Kornblum (Ed.), Attention and performance IV (pp. 629-685). New York, NY: Academic Press.

Sternberg, S., Knoll, R. L., \& Gates, B. A. (1971). Prior entry reexamined: Effect of attentional bias on order perception. Paper presented at the meeting of the Psychonomic Society, St. Louis, MO.

Stevens, S. (1966). Duration, luminance, and the brightness exponent Attention, Perception, \& Psychophysics, 1(2), 96-100.

Stone, J., Hunkin, N., Porrill, J., Wood, R., Keeler, V., Beanland, M., . . . Porter, N. (2001). When is now? Perception of simultaneity. Proceedings of the Royal Society of London B: Biological Sciences, 268(1462), 31-38.

Titchener, E. B. (1908). Lectures on the elementary psychology of feeling and attention. New York, NY: Macmillan. 
Tse, P. U. (2010). Attention underlies subjective temporal expansion. In A. C. Nobre \& J. T. Coull (Eds.), Attention and time (pp. 137-150). Oxford, England: Oxford University Press.

Tse, P. U., Intriligator, J., Rivest, J., \& Cavanagh, P. (2004). Attention and the subjective expansion of time. Attention, Perception, \& Psychophysics, 66(7), 1171-1189.

Tucker, M., \& Ellis, R. (2004). Action priming by briefly presented objects. Acta Psychologica, 116(2), 185-203. https://doi.org/10.1016/ j.actpsy.2004.01.004

Ulrich, R., Nitschke, J., \& Rammsayer, T. (2006). Perceived duration of expected and unexpected stimuli. Psychological Research, 70(2), 77-87. https://doi.org/10.1007/s00426-004-0195-4

Vangkilde, S., Coull, J. T., \& Bundesen, C. (2012). Great expectations: Temporal expectation modulates perceptual processing speed. Journal of Experimental Psychology: Human Perception and Performance, 38(5), 1183.

Vangkilde, S., Petersen, A., \& Bundesen, C. (2013). Temporal expectancy in the context of a theory of visual attention. Philosophical Transactions of the Royal Society B: Biological Sciences, 368(1628), 20130054.

Wearden, J., \& Bray, S. (2001). Scalar timing without reference memory? Episodic temporal generalization and bisection in humans. The Quarterly Journal of Experimental Psychology: Section B, 54(4), 289-309.

Wehrman, J. J., Wearden, J., \& Sowman, P. (2018). The expected oddball: Effects of implicit and explicit positional expectation on duration perception. Psychological Research, 1-15. Advance online publication. https://doi.org/10.1007/s00426-018-1093-5

Weiß, K., \& Scharlau, I. (2011). Simultaneity and temporal order perception: Different sides of the same coin? Evidence from a visual prior- entry study. Quarterly Journal of Experimental Psychology, 64(2), 394-416. https://doi.org/10.1080/17470218.2010.495783

Welch, R. B., \& Warren, D. H. (1980). Immediate perceptual response to intersensory discrepancy. Psychological Bulletin, 88(3), 638.

Yarrow, K. (2018). Timing and time perception: Procedures, measures, and qpplications. In A. Vatakis, F. Balc1, M. Di Luca, \& Á. Correa (Eds.), Collecting and interpreting judgments about perceived simultaneity: A model-fitting tutorial (pp. 295-325). Leiden, Netherlands: Brill. https://doi.org/10.1163/9789004280205

Yarrow, K., Martin, S. E., Di Costa, S., Solomon, J. A., \& Arnold, D. H. (2016). A roving dual-presentation simultaneity-judgment task to estimate the point of subjective simultaneity. Frontiers in Psychology, 7, 416.

Yarrow, K., Whiteley, L., Haggard, P., \& Rothwell, J. C. (2006). Biases in the perceived timing of perisaccadic perceptual and motor events. Perception \& Psychophysics, 68(7), 1217-1226. https://doi.org/10. 3758/BF03193722

Yates, M. J., \& Nicholls, M. E. (2009). Somatosensory prior entry. Attention, Perception, \& Psychophysics, 71(4), 847-859.

Yates, M. J., \& Nicholls, M. E. (2011). Somatosensory prior entry assessed with temporal order judgments and simultaneity judgments. Attention, Perception, \& Psychophysics, 73(5), 1586-1603.

Zampini, M., Shore, D. I., \& Spence, C. (2005). Audiovisual prior entry. Neuroscience Letters, 381(3), 217-222. https://doi.org/10.1016/j. neulet.2005.01.085

Publisher's note Springer Nature remains neutral with regard to jurisdictional claims in published maps and institutional affiliations. 